\title{
Electron temperature measurement of cold plasma in a strong magnetic field
}

\author{
A Dorelon $\uparrow$, M Lombardi $†$ and J C Maan \\ $\div$ Laboratoire de Spectrométrie Physique. BP 53 X. 38041 Grenoble Cedex. France \\ Max Planck Institute BP 166 X, 38042 Grenoble Cedex. France
}

Received 29 April 1981. in final form 13 August 1981

\begin{abstract}
This paper shows how electron temperature and density in low pressure cold plasma $\left(T_{\mathrm{e}}<10 \mathrm{eV}\right)$ can be measured directly from cyclotron resonance absorption measurements in a strong homogeneous magnetic field $(=20 \mathrm{~T})$. For pressures below $0.5 \mathrm{Torr}$, the cyclotron line width is Doppler broadened and the electron temperature is directly derived from this parameter. At high pressures the line width becomes collision broadened and the electron temperature is indirectly derived using the known energy dependence of the collision frequency.
\end{abstract}

\section{Introduction}

Cold plasmas subjected to a strong magnetic field (up to $20 \mathrm{~T}$ ) offer the possibility of interesting studies, such as the relaxation of Rydberg states by electron collisions, using anticrossing spectroscopy techniques (Freund et al 1977). This type of experiment must be performed in a gas pressure range of $10^{-2}$ to a few Torr and in an electron density range $10^{15}-10^{18} \mathrm{~m}^{-3}$. These conditions can be obtained in a high frequency discharge, where the electron temperature is a few $\mathrm{eV}$.

The determination of electron densities and temperature is important in order to analyse experimental results quantitatively. Electron density can be determined by the microwave technique (resonance cavity), even with a magnetic field. if the resonance mode has its electric field parallel to the magnetic field (Golant 1961). On the other hand, electron temperature is more difficult to measure because the few proven methods, such as Langmuir probes or spectroscopic analysis of light (Allis 1981), cannot be applied in this range of pressure and magnetic field.

The measurement of the cyclotron radiation spectrum is widely used for the determination of electron temperature for hot plasmas $\left(T_{\mathrm{c}}>100 \mathrm{eV}\right)$ using the Doppler line broadening technique. Difficulties arise in using the same technique for cold plasmas because the radiated power is low and a very narrow line has to be detected with high resolution. In addition, hypotheses must be made to take into account the self-absorption of radiation.

Cold plasma cyclotron radiation has been extensively studied (Bekefi 1966), but no direct measurement of temperature by Doppler broadening has been carried out because the line shape was determined by collision broadening in the pressure and magnetic field range of the experiments. 
Far-infrared lasers (Hodges 1978) have made new measurements in plasmas possible (Holzauer 1976). The available far-infrared radiation (FIR) frequencies (millimetre and submillimetre waves) are ideally suited to cyclotron resonance experiments in continuous strong magnetic fields ( Lax 1979).

The purpose of this paper is to demonstrate the utility of electron temperature measurements by cyclotron absorption in a strong magnetic field; The final aim is to measure the electron distribution function and densities by this simple nonperturbing method.

\section{Principle of the experiment}

Cyclotron absorption of plasma electrons is measured by means of an electromagnetic wave propagating parallel to the magnetic field direction (Faraday configuration). In this case, the Doppler effect and collisions are the main contributions to the cyclotron resonance (CR) line width.

CR absorption occurs when $\omega=\omega_{c}\left[1-\left(v_{z} / c\right)\right]$, where $\omega / 2 \pi$ is the wave frequency, $\omega_{\mathrm{c}} / 2 \pi$ the cyclotron frequency, $c$ the speed of light and $v_{z}$ the velocity of the electron in the magnetic field $B$. The cyclotron frequency is shifted by the Doppler effect. When this effect is dominant, the distribution function $f\left(v_{z}\right)$ of the electrons along $B$ can be determined from the $C R$ line shape. More specifically, if the distribution is a Maxwell function, the absorption is in the form of a Gauss curve, and the relative half-width $\Delta B / B$ can be used to determine the most probable speed $v_{\mathrm{e}}$ of the electrons.

$$
\frac{\Delta B}{B}=(\ln 2)^{1 / 2} \frac{v_{\mathrm{e}}}{c} \text {. }
$$

The effect is minor for cold plasmas; the relative half-width is $1.6 \times 10^{-3}$ for electrons with energy $1 \mathrm{eV}$.

When collision with atoms is the dominant broadening mechanism, the Doppler effect can be ignored. In this case, if the electron distribution function is isotropic, it has been shown (Bekefi 1966) that the absorption coefficient per unit length $\alpha\left(\omega_{c}\right)$ is given by:

$$
\alpha\left(\omega_{\mathrm{c}}\right)=-\frac{4 \Pi}{3} \frac{\omega_{\mathrm{p}}^{2}}{c} \int_{0}^{\infty} \frac{\nu_{(v)}}{\left(\omega-\omega_{\mathrm{c}}\right)^{2}+\nu_{(v)}^{2}} \frac{\delta f_{(v)}}{\delta v} v^{3} \mathrm{~d} v
$$

where $f(v)$ is the electron distribution function, $\omega_{\mathbf{p}} / 2 \pi$ the plasma frequency and $\nu_{(v)}$ the frequency of collisions with atoms. In this formula, the derivative of the distribution function arises because stimulated emission is taken into account. An alternative form of $\nu_{(v)}$ is $\nu=\sigma_{(v)} N v$, where $\sigma$ is the cross-section and $N$ the atom density. Note that when $\nu$ does not depend on the speed of the electrons, the absorption line is a Lorentz curve in which the half-width $\nu$ depends only on the gas pressure.

Collision broadening is the principal effect in the experiments which use centimetre waves, i.e., magnetic fields of a few tenths of a tesla (Hirshfield and Brown 1961). Since collision broadening affects the absolute width of the line $\Delta B$, and Doppler broadening the relative width $\Delta B / B$ of the line, it is clear that Doppler broadening is most easily observed in strong magnetic fields.

In addition to Doppler and collision broadening, the transit time of the electrons can also contribute to the line shape. If $v$ is the speed of the electron and $l$ the characteristic length of the measurement (here $l$ is approximately the length of the plasma slab), the 
broadening produced by this effect is proportional to $v / 2 \pi l$ and Doppler broadening to $v / \lambda$, where $\lambda$ is the wavelength. Since here $\lambda \simeq 1 \mathrm{~mm}$ and $l \simeq 10 \mathrm{~mm}$, this transit time broadening is two orders of magnitude smaller than the Doppler broadening. An additional advantage of the use of strong magnetic fields is that $\omega_{c} \gg \omega_{p}$ so that dispersion effects of a wave propagating in a plasma (French et al 1961) can be neglected.

In conclusion, in the present plasma conditions, Doppler broadening is expected to be greater than collision broadening for the lower pressure used. To show experimentally that Doppler broadening is dominant, the following conditions should be fulfilled:

(i) absorption half-width does not extrapolate to zero for decreasing pressure;

(ii) the relative half-width $\Delta B / B$ is frequency independent;

(iii) for a pure maxwellian distribution function, the shape of the line becomes gaussian at low pressure.

\section{Experimental details}

The FIR transmission of the plasma as a function of the magnetic field was measured using the set-up shown in figure $1(a)$. The plasma was generated under steady-state conditions in a quartz cell placed in the centre of a Bitter coil (figure 1(b)) that can produce fields of up to $19 \mathrm{~T}$ with a field homogeneity of $2 \times 10^{-4}$ in the plasma volume.

The $10 \mathrm{~mm}$ thick, $20 \mathrm{~mm}$ diameter plasma slab was produced by the electric field of two plates of a condenser fed by a $60 \mathrm{MHz}$ AC voltage. The voltage necessary to break down and sustain this discharge was obtained by a quarter-wave coaxial line that acted as a transformer powered by a $3 \mathrm{~W}$ radiofrequency generator.

The FIR, generated by an alcohol-filled laser (1995, Edinburgh Instruments), pumped by a $30 \mathrm{~W}, \mathrm{PL3}, \mathrm{CO}_{2}$ laser (also from Edinburgh Instruments), was guided by an overmoded waveguide and focused to a $5 \mathrm{~mm}$ diameter spot in the plasma.

The FIR was detected by a bolometer (Infrared Laboratories Inc.). A mirror mounted on a rotating wheel transmitted alternately (frequency $20 \mathrm{~Hz}$ ) the FIR power to the plasma and to a reference detector. The output of two lock-in detectors monitoring the measurement and the reference signals was divided by a PAR model 188 ratiometer to cancel the fluctuations in laser intensity. The overall noise was less than $2 \%$. FIR power (less than $1 \mu \mathrm{W}$ ) was low enough for the plasma not to be heated; this was confirmed by monitoring the total undispersed light emitted by the plasma with a 1 P 28 (Hamamatsu) photomultiplier.

In the measurements an error may arise because, in the interacting zone of the FIR with the plasma, the electromagnetic wave propagating at a non-zero angle with respect to the magnetic field reduces the Doppler broadening. However, for the apertures of our experiment, this would result in an error of less than $3 \%$. Interference effects due to multiple reflections (French et al 1961) of FlR against condenser plates were avoided by an antireflection coating.

\section{Results}

The $C R$ absorption was measured as a function of the magnetic field and pressure in a plasma generated in a high purity gas (impurities 5 PPM). Helium and argon were the gases used in the experiments. Argon was selected because it is easily ionised, thereby allowing lower experimental pressures to be used. Two wavelengths. $1.223 \mathrm{~mm}$ and 

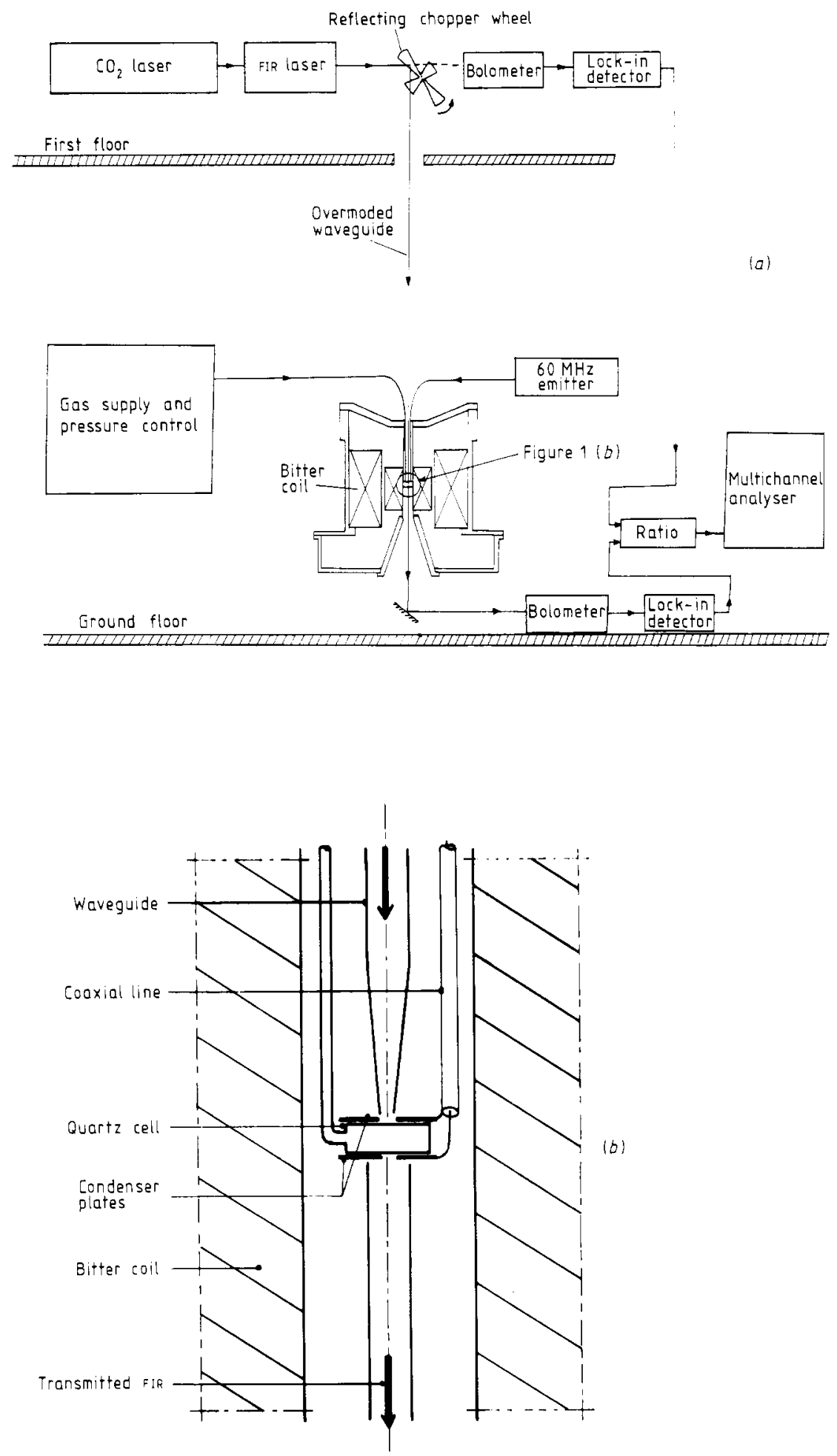

Figure 1. (a) General view of experimental set-up. (b) Plasma slab set-up. 
$0.6994 \mathrm{~mm}$, were used to check the frequency dependence. CR occurs at $8.75 \mathrm{~T}$ and $15.32 \mathrm{~T}$ respectively, which is in good agreement with the theoretical $\mathrm{CR}$ of an electron in the vacuum. Figure 2 shows experimental results for helium and figure 3 for argon.

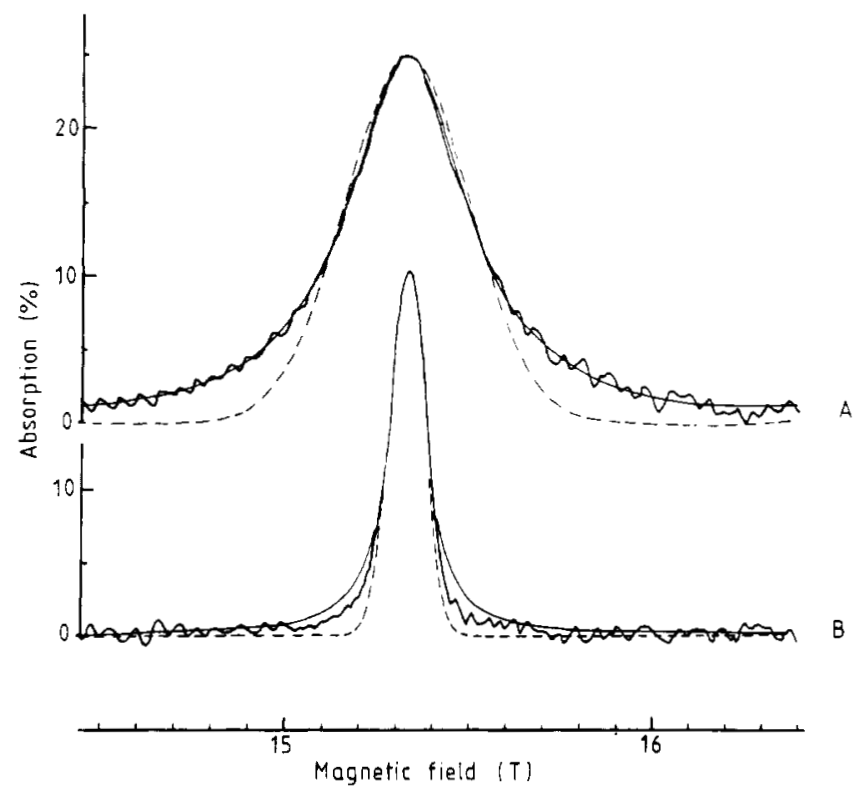

Figure 2. Cyclotron absorption versus magnetic field in helium. FIR wavelength: $0.6994 \mathrm{~mm}$ at $P=21$ Torr (curves $A$ ) and $P=1.0$ Torr (curves $B$ ). Full curve with fluctuations. experimental results: smooth full curve. Lorentz curve: broken curve, Gauss curve.

\subsection{Half-width of curves}

The absorption rates measured in these experiments varied from a few percent to $70 \%$ of the $C R$ active polarisation, according to pressure and discharge conditions. The results for the half-width presented are corrected by calculating the absorption coefficient from the transmitted intensity. Figure 4 shows our results of the half-width, as a function of helium pressure.

At high pressure, the half-width of the absorption curves increases with pressure, and the measurements taken with both wavelengths have an identical half-width. This result obtained both with helium and argon indicates that collision broadening is dominant. For helium, figure 4 shows that the half-width tends to a constant value when the pressure decreases. Indeed, when extrapolated to zero pressure, the half-width $\Delta B$ is $0.04 \mathrm{~T}$ for the $1.223 \mathrm{~mm}$ wavelength and $0.065 \mathrm{~T}$ for the $0.6994 \mathrm{~mm}$ wavelength. These half-widths are greater than the non-homogeneity in the magnetic field which is $0.0017 \mathrm{~T}$ and $0.0030 \mathrm{~T}$ respectively; this result shows that the zero pressure extrapolated halfwidth is not zero, but is one order of magnitude greater than the magnetic field nonhomogeneity.

At the lowest working pressure ( 0.5 Torr) in helium, the ratio of the half-widths for the two wavelengths $(1.70 \pm 0.08)$ agrees favourably with the theoretical inverse ratio of the wavelengths (1.749). For each of these two measurements, the relative half-width $\Delta B / B$ is $4.5 \%$ and this good agreement proves that the Doppler effect is the dominant cause of the line broadening. Using equation (1), an electron temperature of $7.5 \mathrm{eV}$ is 


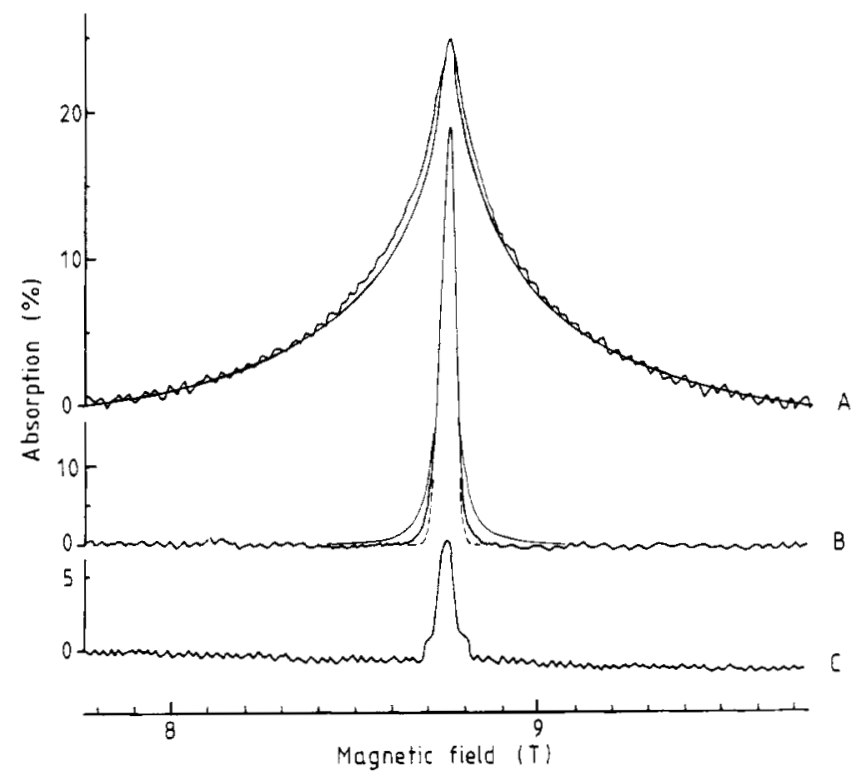

Figure 3. Cyclotron absorption versus magnetic field in argon. FIR wavelength: $1.223 \mathrm{~mm}$. Curves A: Full curve with fluctuations, experimental results: $P=18.5$ Torr. Smooth full curve, computed results with collision broadening (electron temperature $2.5 \mathrm{eV}$ ). Curves $\mathrm{B}$ : Full curve with fluctuations, experımental results: $P=0.20$ Torr. Smooth full curve. Lorentz curve: broken curve, Gauss curve. Curves A and B were taken with 3 W RF power. Curve C: Experimental results: $P=0.030$ Torr; $10 \mathrm{~W}$ RF power.

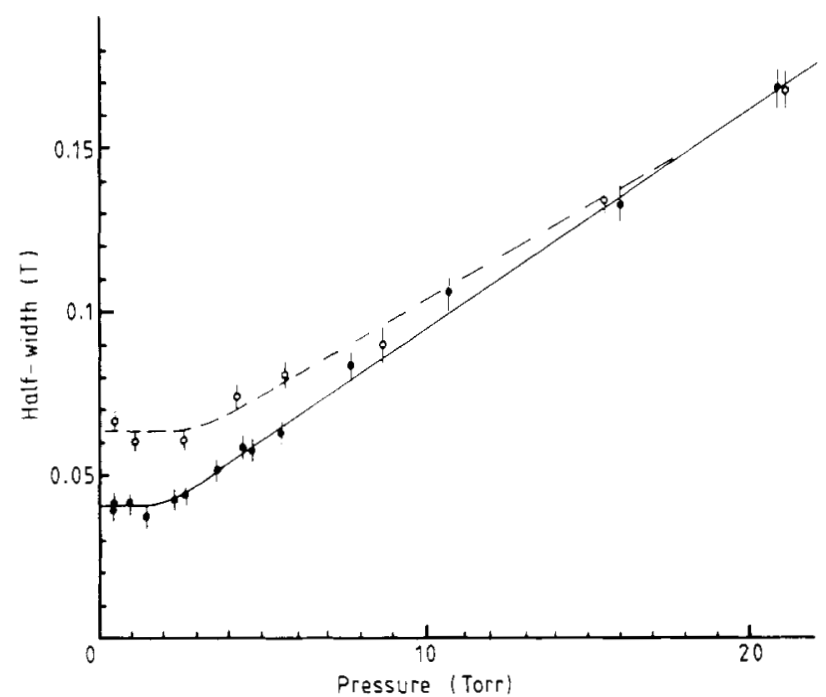

Figure 4. Helium: half-width at half-height of absorption coefficient $\alpha$ as a function of pressure. $\lambda=1.223 \mathrm{~mm} ; 0, \lambda=0.6994 \mathrm{~mm}$.

obtained with a $10 \%$ accuracy. For higher pressures, the relative half-widths measured with the two wavelengths are no longer the same since collision broadening contributes to the line width.

For argon, the same conclusions were reached: the extrapolation of the half-width to zero pressure is not zero and the extrapolated value is greater than the field non- 
homogeneity. Since the discharge can be maintained at low pressure, the more interesting results relative to the Doppler effect are obtained in the pressure range $0.03-$ 2 Torr, and are given in figure 5 for both wavelengths.

For a better presentation of the Doppler broadening, the relative half width $\Delta B / B$ of the curves is given as a function of pressure. When the pressure is lower than 0.5 Torr, the results obtained with the two wavelengths are the same. This agreement shows that the relative half-width is frequency independent. According to relation (1). this is proof that the Doppler effect is dominant in the broadening of the CR absorption. With a relative half-width of $2.4 \%$, an electron temperature of $2.2 \mathrm{eV}$ is obtained with an accuracy of $10 \%$.

In conclusion, at low pressures, both the pressure and the frequency dependence of the half-width clearly show the expected behaviour for a Doppler broadened line width.

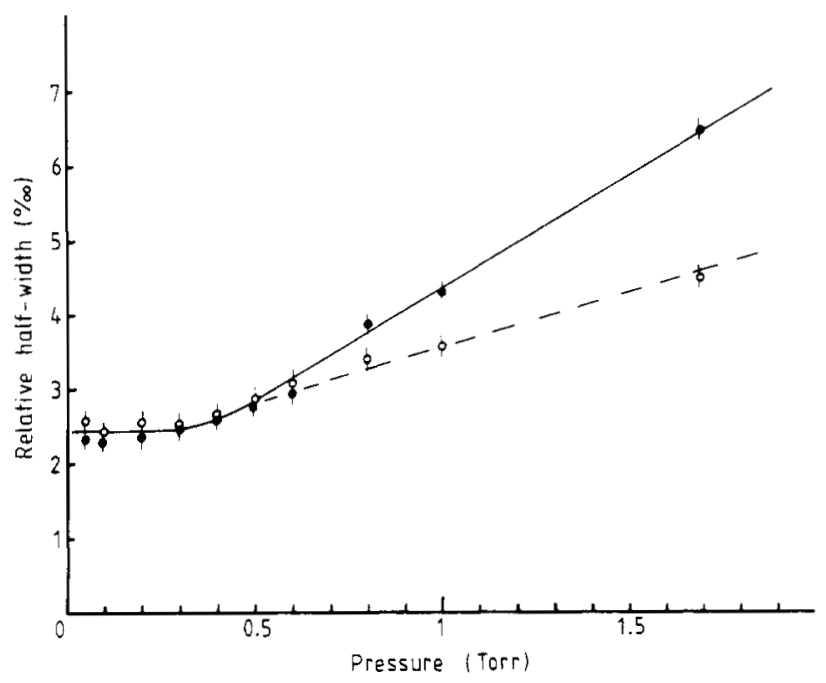

Figure 5. Argon: relative half-width of absorption coefficient $\alpha$ as a function of pressure (low pressure range). $\lambda=1.223 \mathrm{~mm}: 0 . \lambda=0.6994 \mathrm{~mm}$.

\subsection{Line shape}

The line shape at low pressure (Doppler broadening) and high pressure (collision broadening) is now analysed in greater detail.

In figure 2, curves B show the line profile for helium at 1 Torr. Lorentz and Gauss curves with the same height and half-width are given for comparison. The experimental data give a curve which lies in between the Lorentz and Gauss curves.

In figure 3 , curves $B$ show the measurement for argon at 0.2 Torr and the same conclusions are found as for helium. The shape of the curve becomes gaussian. More precisely, within this pressure range the line has a Voigt shape (Posener 1959), i.e. in the centre of the line, Doppler broadening is dominant but, because of the very rapid decrease of the Gauss function on the wings, collision broadening still plays a role in the line for this pressure. To obtain a pure gaussian profile the pressure should be even lower. Unfortunately, the signal becomes too small to be measured accurately under such conditions. 
The preceding experiments were performed with a $3 \mathrm{~W}$ radio frequency power as reported. With both gases, in the low pressure zone, when radio frequency power increases, the absorption curves are broadened, indicating an increase in plasma temperature. For argon, when the pressure is low enough $\left(3 \times 10^{-2}\right.$ Torr $)$, and when the power is increased to its maximum $(10 \mathrm{~W})$, the shape of the absorption line is modified as seen in figure 3 , curve $C$. Two shoulders appear on the edge of the line, symmetrical with respect to the centre of the line and with a sharp cut-off.

For such pressures, the electron-atom collision frequency is of the same order of magnitude as the discharge frequency, implying that electrons are accelerated by the electric field along the magnetic field and not completely thermalised by collisions. The electrons partly follow the sinusoidal electric field excitation and their speed has a sinusoidal component with a maximum leading to a characteristic cut-off. This can explain the deviations from a gaussian line shape. When the radio frequency power is increased, the maximum speed corresponding to the sinusoidal component is observed on the edge of the absorption line. This method can thus provide information on the electron distribution function.

As previously mentioned, at high pressure the line shape is broadened by collision. Consequently, the collision frequency must be considered in the analysis of the high pressure data. At microscopic level, the collision cross-section for electrons with helium atoms is nearly energy-independent in the low energy $(0-5 \mathrm{eV})$ range and decreases for higher energy (Laborie et al 1968). A constant cross-section is equivalent to a constant mean free path for electrons. The collision probability of $20 \mathrm{~cm}^{-1} \mathrm{Torr}^{-1}$ is reported by Hirshfield and Brown (1958), for low energy electrons in helium.

The collision frequency $\nu=1.19 \times 10^{9} \sqrt{V} P$ where $\nu$ is in $\mathrm{Hz}, P$ in Torr and $V$ in eV is deduced for low energies. Taking into account the collision frequency and assuming a Maxwell electron velocity distribution, the absorption line is calculated as a function of $B$ using relation ( 2 ) for collision broadening.

No significant difference was found between constant mean free path and Lorentz line shape calculation. The same observation was made by Kelly et al (1957) in theoretical analysis. In the constant mean free path model, the half-width depends on the mean electron energy and the electron temperature can be determined by comparing experimental data and calculations. In figure 2, curves A show a comparison between theoretical Lorentz and Gauss curves with same height and half-width as the experimental curve. The agreement between the Lorentz curve and the experimental curve is good for an electron temperature of $1 \mathrm{eV}$ and an electron density of $9.4 \times 10^{16} \mathrm{~m}^{-3}$.

With argon, the curves in the high pressure range are very pointed and do not correspond to a Lorentz curve as seen in figure 3, curves A. Such curve shapes were reported in cyclotron emission by Wright and Bekefi (1971). The cross-section of electrons colliding with argon atoms increases with energy for energies lower than $10 \mathrm{eV}$ (Laborie et al 1968) and is explained by the Ramsauer effect. Within this range, the collision frequency $\nu=3.96 \times 10^{8} V^{3 / 2} P$, can be deduced from available cross-sections. Using this dependence in equation (2), and assuming a maxwellian electron speed distribution, theoretical absorption curves were calculated and are given in figure 3 (curves A).

The curve shape resulting from this calculation is in good agreement with the experimental shape. For a given pressure, the half-width of the curves depends on the electron temperature, thereby enabling this temperature to be deduced by comparison with the experimental results. In figure 3, curves A, a typical calculation result is shown. An electron temperature of $2.5 \mathrm{eV}$ in the calculation and an electron density of 
$1.3 \times 10^{17} \mathrm{~m}^{-3}$, give the best agreement between calculated and measured line shapes. On the wings, theoretical curves closely fit experimental curves. At the centre of the line, where the line is narrow, the theoretical curve is narrower than the experimental one. This may be due to Doppler broadening which is neglected in this calculation.

In conclusion, when collision broadening is dominant, taking into account the electron cross-section, the electron temperature can still be deduced from line shape but this method is more indirect than that using the line shape in the Doppler regime.

\section{Conclusions}

This experiment shows that electron temperature can be measured in a cold plasma submitted to a strong magnetic field and far-infrared radiation using the cyclotron resonance line width.

In the low pressure range, Doppler broadening is dominant and the line shape gives direct information on the electron speed. In the high pressure range, collision broadening is dominant and the electron temperature can be measured using the cross-section of the gas. In this case, the electron temperature is obtained more indirectly and therefore less accurately than in the Doppler regime.

\section{Acknowledgments}

The experiments were carried out at the Service National des Champs Intenses in Grenoble, a joint facility of the CNRS and the MPI. We would like to acknowledge the help of Dr Schneider Muntau in the design of the homogeneous Bitter coil and of $\mathrm{Mr}$ and Mrs G A Sumner for reviewing, correcting and typing the English text.

\section{Addendum}

Effective collision frequencies of electrons in noble gases are given by Baille (1981) with greater details than the formulae used in this article. But such refinements are not critical in the computation of the broadened line shape.

\section{References}

Allis W P 1981 Arc Discharge and Electrical Discharge (Les Arcs, France: NATO Advanced Study Institute) Baille P 1981 J. Phys. B. At. Mol. Phys. $141485-95$

Bekefi G 1966 Radiation Processes in Plasmas (New York: Wiley)

French I P, Cloutier G C and Bechynski M P 1961 Can. J. Phys. 39 1273-90

Freund R S, Miller T A, Zegarski B R, Jost R, Lombardi M and Dorelon A 1977 Chem. Phys. Lett. 51 18-22 Golant V E 1961 Sov. Phys.-Tech, Phys. 5 1197-310

Hirshfield J L and Brown S C 1958 J. Appl. Phys. 29 1749-52

- 1961 Phys. Rev. 122 719-25

Hodges D T 1978 Infrared Phys. 18375

Holzhauer E 1976 Infrared Phys. 16 135-43

Kelly D C, Margenau H and Brown S C 1957 Phys. Rev. 108 1367-71 
Laborie P, Rocard J and Rees $\mathrm{J} 1968$ Electronic cross-section and macroscopic coefficients: hydrogen and rare gases (Paris: Dunod)

Lax B 1979 J. Magn. Magnetic Mater. 11 1-15

Posener D W 1959 Aust. J. Phys. $12184-96$

Wright B L and Bekefi G 1971 Phys. Fluids 14 1773-81 